\title{
The reflection coefficients of multilayer anti-counterfeiting optical films
}

\section{for S-polarized wave}

\author{
Hao Yannan, Liu Fuping, Xu Yue , Wang Anling \\ (Beijing Institute of Graphic Communication, Beijing, 102600, China. ) \\ afupingliu60@sina.com (corresponding author),bwanganling@bigc.edu.cn
}

\begin{abstract}
Key words Multilayer anti-counterfeiting optical film, reflection coefficients of light, S-polarized wave
\end{abstract}

Abstract Considering the difference of phase-shift direction and amplitude attenuation direction when the optical wave propagates in conductive medium, with the boundary conditions of optical wave at the interface of the media, we derive the recurrence formula reflection coefficient for the S-polarized optical wave in the multilayer film. Given a calculating example for multilayer anti-counterfeiting optical films, the result shows that thickness of metal film has important effect on the reflection coefficient of light, and the multilayer film has stronger reflection effective of narrow band.

\section{Introduction}

Color anti-counterfeiting printing ink has gradually become an important part of modern social security field and means ${ }^{[1-2]}$, because of it has dynamic color changing effect, has very good concealment, can be clearly seen when the view angle changes the color changed of original design image, with good intuitive that can identify with the eye ${ }^{[3-4]}$, color copiers and electronic scanners cannot copy it. For printing ink manufacturing complex, difficult to counterfeit, so color changing ink anti-counterfeit printing technology in cash, check, high securities demanding such as bonds, stocks has important application. In this kind of membrane system contains high conductivity of metal film. In most cases, it is different between the direction of amplitude attenuation and phase shift spreading direction. Although the anti-counterfeiting film system in using today the metal film is very thin $(2-8 \mathrm{~nm})$, it have difference huge scale between the metal film's conductivity and dielectric film's conductivity (in the $10^{10}$ order of magnitude) ${ }^{[5-6]}$, so the metal film have big effects for optical propagation spread in the film. But the current literatures that have seen are mostly a simplified treatment to the metal film. it will be bring bigger error for calculation accuracy. In this article, we will consider the propagation characteristics of the general inhomogeneous wave, and use the light wave propagation equation of multilayer film's membrane system to give computing an example for S-polarized wave, and get some new rule understanding.

\section{The inhomogeneous wave's Snell Formula}

Because the direction of phase constant $\alpha$ and amplitude attenuation constant $\beta$ is not consistent, we adopt the method of figure 1 to representation the direction relations of $\alpha$ and $\beta^{[6]}$, whose space propagation waves at point A, B, C and D.The complex wave vectors of the incident, 
reflected and refracted waves can be written as $\boldsymbol{k}_{1}=\boldsymbol{\alpha}_{1}-\mathrm{i} \boldsymbol{\beta}_{1}, \boldsymbol{k}_{r}=\boldsymbol{\alpha}_{r}-\mathrm{i} \boldsymbol{\beta}_{r}, \boldsymbol{k}_{2}=\boldsymbol{\alpha}_{2}-\mathrm{i} \boldsymbol{\beta}_{2}$, where, i is imaginary unit. According to the continuous condition of the electromagnetic field we have $\alpha_{1} \sin \psi_{1}=\alpha_{2} \sin \psi_{2}, \beta_{1} \sin \varphi_{1}=\beta_{2} \sin \varphi_{2}$.
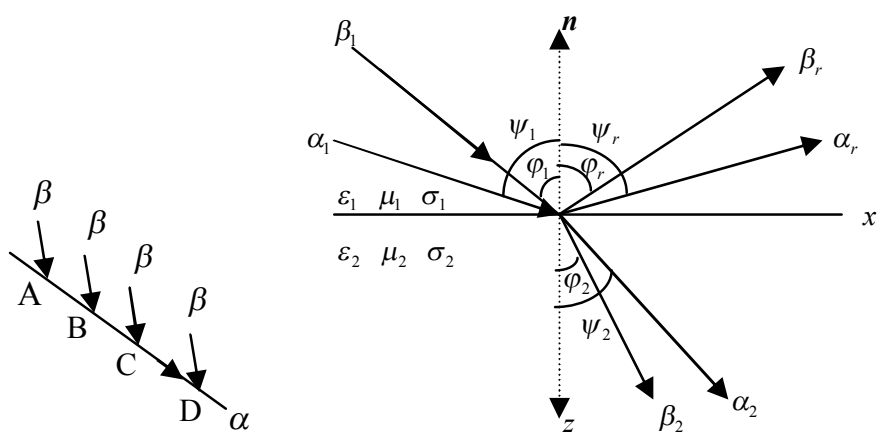

Fig.1 The reflection and refraction of inhomogeneous optical wave at an interface between two conductive media

\section{The field distribution of S-polarized wave in the multilayer optical films}

Set the $\mathrm{E}$ and $\mathrm{H}$ respectively as the electric field and magnetic field of light waves, the electromagnetic field in the $\mathrm{j}$-th layer can be written as

$$
\boldsymbol{E}_{j}=\boldsymbol{E}_{j}^{+} e^{-i \boldsymbol{k}_{j}^{+} \cdot \boldsymbol{r}}+\boldsymbol{E}_{j}^{-} e^{-i \boldsymbol{k}_{j}^{-} \cdot \boldsymbol{r}}, \boldsymbol{H}_{j}=\boldsymbol{H}_{j}^{+} e^{-i \boldsymbol{k}_{j}^{+} \cdot \boldsymbol{r}}+\boldsymbol{H}_{j}^{-} e^{-i \boldsymbol{k}_{j}^{-} \cdot \boldsymbol{r}}
$$

Where, $k_{j}$ is the complex wave vector of light in the $\mathrm{j}$-th layer of the conductive medium. The relationships between $E$ and $H$ are

$$
\boldsymbol{H}_{j}^{+}=\frac{1}{k_{j}} \sqrt{\frac{\varepsilon_{j}}{\mu_{j}}} \boldsymbol{k}_{j}^{+} \times \boldsymbol{E}_{j}^{+}, \quad \boldsymbol{H}_{j}^{-}=\frac{1}{k_{j}} \sqrt{\frac{\varepsilon_{j}}{\mu_{j}}} \boldsymbol{k}_{j}^{-} \times \boldsymbol{E}_{j}^{-},
$$

By light wave continuity conditions of the electromagnetic field we have

$$
\begin{gathered}
M_{i}\left(\begin{array}{c}
E_{j}^{+} \\
E_{j}^{-}
\end{array}\right)=N_{j}\left(\begin{array}{c}
E_{j+1}^{+} \\
E_{j+1}^{-}
\end{array}\right), \\
M_{j}=\left[\begin{array}{cc}
e^{-i k_{j z} z_{j}} & e^{i k_{j z} z_{j}} \\
\eta_{j} e^{-i k_{j z} z_{j}} & -\eta_{j} e^{i k_{j z} z_{j}}
\end{array}\right], \quad N_{j}=\left[\begin{array}{cc}
e^{-i k_{j+1 z} z_{j}} & e^{i k_{j+1} z_{j}} \\
\eta_{j+1} e^{-i k_{j+1 z} z_{j}} & -\eta_{j+1} e^{i k_{j+1 z} z_{j}}
\end{array}\right],
\end{gathered}
$$

If the optical wave membrane has $J+1$ layers, then $E_{J+1}^{+}=0$, with above relations we can get the field distribution of the multilayer films, thus we can get the total amplitude reflection coefficient and total energy reflectivity $r=\frac{E_{1}^{-}}{E_{1}^{+}}$and $R=|r|^{2}$.

\section{The example analysis}

The following membrane system is $\mathrm{A} / y \mathrm{M} 0.93 \mathrm{~L}(\mathrm{LH})^{2}(\mathrm{LH})^{2} 0.76 \mathrm{~L} 100 \mathrm{~N} / \mathrm{G}$, where A medium is one of the most top, $\mathrm{G}$ is the lowest medium, $\mathrm{M}$ represents a chromium film, $\mathrm{N}$ denotes the silver 
film, $\mathrm{L}$ and $\mathrm{H}$ are the low and high refractive index dielectric film,In the membrane system, $n_{H}=2.35, n_{L}=1.32, P=2, n_{g}=1.52, n_{A}=1.52$, Chromium film thickness is $\mathrm{y}=2,4,7 \mathrm{~nm}$, the film thickness of $\mathrm{Ag}$ is $100 \mathrm{~nm}$, center wavelength is $520 \mathrm{~nm}$, the conductivity of chromium film is $\sigma=7.752 \times 10^{6}$, the conductivity of the silver film is $\sigma=6.06 \times 10^{7}$, complex refractive indexes of Chromium and Silver film $n_{c r}=2.91-3.33 \mathrm{i}$ and $n_{a g}=0.13-3.07 \mathrm{i}$. From the figure 3 we can see that the amplitude of the reflection coefficient is decreasing with the increasing film thickness of metallic chromium, which is actually due to the increased absorption capacity of the chromium-based film is enhanced due to the film thickness.

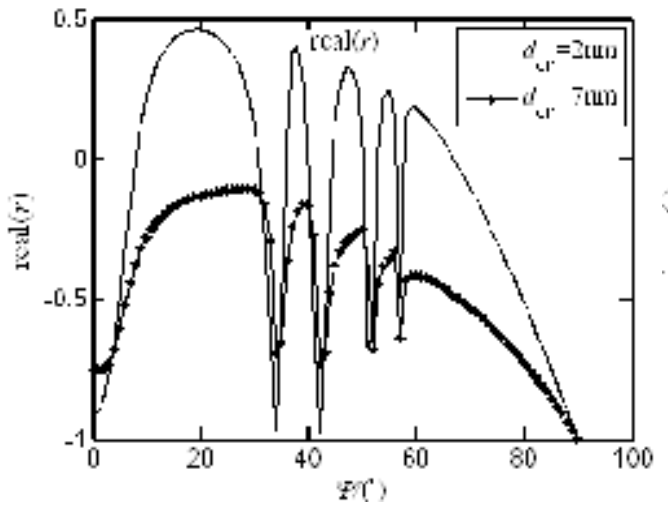

Fig. 2 The reflection coefficient (imaginary Fig.3 The reflection coefficient part)of light of multilayer film

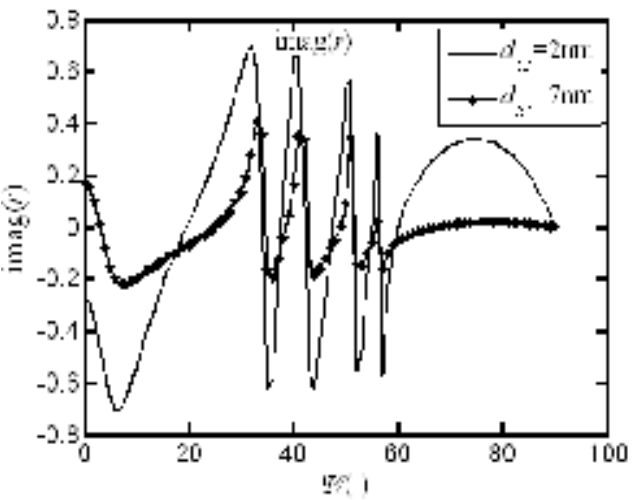
(real part) of multilayer film

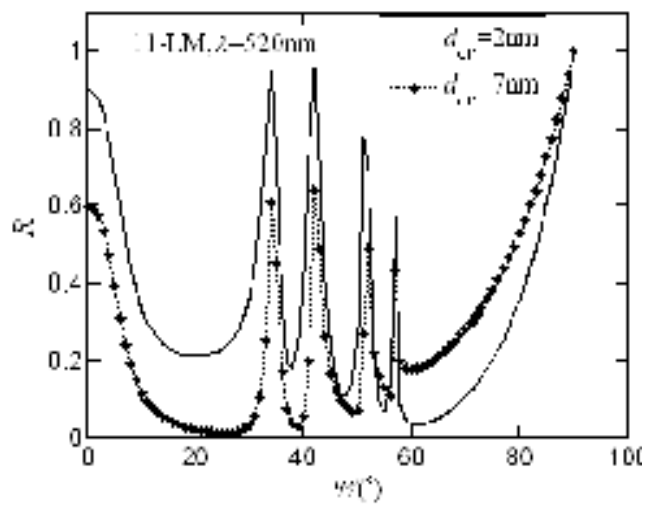

Fig.4 The energy reflectivity of multilayer film varying with incidence angle

From Figure 4 can also be found that the size of imaginary part of the reflection coefficient curve is decreasing with the chromium film thickness increasing, which is due to the presence of the metal film, the size of the imaginary part of the reflection coefficient curves accurately reflect the absorption effect of the metal film. Figure 5 is the curves of the energy reflectance, where the main wavelength $\lambda=520 \mathrm{~nm}$. This Figure indicates that the total energy reflectivity thereof is gradually reduced with the film thickness of chromium increasing, which reflects the absorbing effect of metal film for light, and the total energy reflectance has a strong narrow band reflection effect. This feature is designed for the security of our narrowband optical zoom high-reflection film is 
meaningful.

\section{Conclusion}

Considering the difference of uneven light's phase shift direction of propagation and its direction of amplitude attenuation. We derived the transitive relation equation of non-uniform S-polarized light waves in multilayer anti-counterfeiting light membrane with boundary conditions of electromagnetic field of light wave. We did not make any approximate reasoning and hypothesis, it closer to the light of the actual wave propagates in conductive medium, so the method has higher accuracy and adaptability should be. The algorithm was realized through actual examples, the case clearly shows that the thickness of the metal film have a great effect on the calculation result is, with the increase of the thickness of metal chromium film reflectivity is diminishing its total energy (which is caused by the absorption characteristics of the metal film). Numerical example also showed that there is a stronger narrowband reflection effect for the optical anti-counterfeiting film. These characteristics for us to design the narrowband and high reflected anti-counterfeiting optical film will have important reference value.

\section{Acknowledgements}

This work was Supported by Supported by the Beijing City Board of Education Science and technology key project (KZ20150015015), Supported by Beijing Natural Science Foundation (4142016) , Supported by the Beijing City Board of Education Science and technology project(KM201510015009), Supported by BIGC Key Project(E-a-2013-19).

\section{Reference}

[1] Zhang J F, Liu L M,Ye Y D. Optical anti-counterfeiting technology and its application(in Chinese). Beijing: National Defense Industry Press,2011 : 175-216

[2] Zhang Y X, Chen J Shi G C. Color Prediction Model of Optically Variable Anti-forgery Ink. Packaging Engineering,2011,32(5): : 94-96

[3] Tan M Q, Lin Y C, Zhao D Z. The properties of periodic symmetrical coatings and the Design of high reflectivity coatings of narrow band for containing the ultra-thin metallic film(n=k) (in Chinese). Acta Photonica Sinica, 1996,25(1):1011-1017.

[4] Mathieu H , Hersch R D. Classical print reflection models: A radiometric approach [ J ] . Imaging S ci T echno ,2004, 48( 4) : $363--373$.

[5] Xu J H. The Electromagnetic Field and Electromagnetic Wave in Layer Media (in Chinese). Beijing: Petroleum Industry Press, 1997: 38 - 59

[6] Liu F P, Wang A L, Chen Q,et al. The research progress on lateral shift of reflected electromagnetic wave at the interface of two conductive media. Chinese Science Bulletin, 2008,53:961-968 